\title{
OPEN MYCN-induced nucleolar stress drives an early senescence-like transcriptional program in hTERT-immortalized RPE cells
}

\author{
Sofia Zanotti ${ }^{1,2,3}$, Suzanne Vanhauwaertt, ${ }^{2,3}$ Christophe Van Neste ${ }^{2,3}$, Volodimir Olexiouk ${ }^{2,3}$, \\ Jolien Van Laere ${ }^{2,3}$, Marlies Verschuuren ${ }^{1}$, Joni Van der Meulen 2,3,4, Liselot M. Mus 2,3,5, \\ Kaat Durinck ${ }^{2,3}$, Laurentijn Tilleman ${ }^{3,6}$, Dieter Deforce ${ }^{3,6}$, Filip Van Nieuwerburgh ${ }^{3,6}$, \\ Michael D. Hogarty ${ }^{7,8}$, Bieke Decaesteker ${ }^{2,3,9}$, Winnok H. De $\operatorname{Vos}^{1,9}$ \& Frank Speleman ${ }^{2,3 凶}$
}

MYCN is an oncogenic driver in neural crest-derived neuroblastoma and medulloblastoma. To better understand the early effects of MYCN activation in a neural-crest lineage context, we profiled the transcriptome of immortalized human retina pigment epithelial cells with inducible MYCN activation. Gene signatures associated with elevated MYC/MYCN activity were induced after $24 \mathrm{~h}$ of MYCN activation, which attenuated but sustained at later time points. Unexpectedly, MYCN activation was accompanied by reduced cell growth. Gene set enrichment analysis revealed a senescencelike signature with strong induction of $\mathrm{p} 53$ and $\mathrm{p} 21$ but in the absence of canonical hallmarks of senescence such as $\beta$-galactosidase positivity, suggesting incomplete cell fate commitment. When scrutinizing the putative drivers of this growth attenuation, differential gene expression analysis identified several regulators of nucleolar stress. This process was also reflected by phenotypic correlates such as cytoplasmic granule accrual and nucleolar coalescence. Hence, we propose that the induction of MYCN congests the translational machinery, causing nucleolar stress and driving cells into a transient pre-senescent state. Our findings shed new light on the early events induced by MYCN activation and may help unravelling which factors are required for cells to tolerate unscheduled MYCN overexpression during early malignant transformation.

MYCN belongs to the MYC protein family of bHLH transcription factors, also including MYC and MYCL. While MYC is commonly activated in many cancers, MYCN is also increasingly recognized as important oncogenic driver in a growing number of cancer entities. MYCN was first discovered in neuroblastoma and later also found to be overexpressed in retinoblastoma, brain tumors, leukemia, neuro-endocrine prostate cancers and pancreatic cancer ${ }^{1}$. MYCN has been extensively studied in neuroblastoma where it marks a subset of high-risk children with poor prognosis despite intensive multi-modal therapy. While mutational burden in these tumors is low, MYCN amplified neuroblastomas and medulloblastoma often exhibit large copy number alterations, most notably $17 \mathrm{q}$ gain $^{2,3}$, indicating that additional gene dosage effects are required to support tumor initiation and/or progression. Given their structural properties, the MYC proteins have been considered as undruggable, but new insights into their function and protein interactions are providing exciting insights towards novel strategies to target these oncogenes. Despite decades of intensive research, the mechanism through which MYC(N) proteins drive cancer formation remain poorly understood. Most of the earlier work focussed on the identification of directly regulated target genes assuming a classical transcription factor role. More recently, evidence was obtained

${ }^{1}$ Laboratory of Cell Biology and Histology, Dept. Veterinary Sciences, University of Antwerp, Universiteitsplein 1, 2610 Antwerp, Belgium. ${ }^{2}$ Center for Medical Genetics, Dept. Biomolecular Medicine, Ghent University, Medical Research Building (MRB), 2nd Floor, Entrance 34, Corneel Heymanslaan 10, 9000 Ghent, Belgium. ${ }^{3}$ Cancer Research Institute Ghent (CRIG), Ghent University, 9000 Ghent, Belgium. ${ }^{4}$ Molecular Diagnostic, Ghent University, 9000 Ghent, Belgium. ${ }^{5}$ Bioresource Center Ghent, Health, Innovation and Research Center, Ghent University, Corneel Heymanslaan 10, 9000 Ghent, Belgium. ' Laboratory of Pharmaceutical Biotechnology, Ghent University, Ottergemsesteenweg 460, 9000 Ghent, Belgium. ${ }^{7}$ Children's Hospital of Philadelphia, Philadelphia, PA 19104, USA. ${ }^{8}$ Perelman School of Medicine, University of Pennsylvania, Philadelphia, PA 19104, USA. ${ }^{9}$ These authors contributed equally: Bieke Decaesteker and Winnok H. De Vos. ${ }^{\varpi}$ email: franki.speleman@ugent.be 
that MYC(N) influences global transcriptional changes through a so-called "amplifier" mode of action ${ }^{4}$. Finally, based on protein-interactions and further in-depth biochemical studies by the Eilers team ${ }^{1}, \mathrm{MYC}(\mathrm{N})$ oncogenicity was attributed to its role in enhancing transcription-stress resilience in tumour cells. This includes roles in alleviating replication fork stalling resulting from nucleotide depletion and POL II stalling, R-loop formation and replication-transcription conflicts ${ }^{5}$. Despite these increasing insights, our current understanding of the early cellular responses that allow cells to cope with the consequence of MYCN overexpression in non-cancerous cells are limited. Hence, we explored the early changes in the dynamic transcriptional landscape of the hTERTimmortalized tamoxifen-inducible RPE1-MYCN-ER human cell model.

In contrast with the known growth promoting effect of MYCN, we observed attenuated cell growth accompanied with nucleolar coalescence, appearance of cytoplasmic granules and increased p21 and p53 protein levels. Yet, senescence hallmarks such as $\beta$-galactosidase positivity, DNA damage or heterochromatin foci formation were absent. We further found that MYCN-induced growth deceleration is accompanied with a transcriptional signature that resembles early senescence induction thus offering a platform for further mechanistic studies to explore cellular processes that protect against early MYCN-induced cellular transformation.

\section{Results}

MYC/MYCN gene signature analysis validates MYCN activation in immortalized RPE1 cells. To study the early effects of MYCN activation, we used 4-OH tamoxifen (4-OHT) inducible MYCN gene regulation in telomerase immortalized RPE1 cells (further referred to as RPE1-MYCN-ER) ${ }^{6}$. In this model, chaperonins persistently sequester the chimeric MYCN-ER protein in the cytosol. The oestrogen receptor ligand 4-OHT will displace the chaperonins, causing MYCN-ER to translocate to the nucleus where it will exert its transcriptional activity. Western blotting confirmed the expected decrease in the cytoplasmic fraction of MYCNER protein (Fig. 1a), and concomitant increase in nuclear levels $24 \mathrm{~h}$ after the addition of 4-OHT to RPE1MYCN-ER cells (MYCN-ON) as compared to mock-treated RPE1-MYCN-ER (MYCN-OFF) and wild type RPE1 (WT) cells (Fig. 1b).

First, we evaluated the functionality of MYCN after nuclear translocation in RPE1 cells by measuring the transcriptional impact. To this end, bulk RNA sequencing was performed at three sequential time points post induction (p.i.) (24 h, $48 \mathrm{~h}, 72 \mathrm{~h}$ ) for MYCN-ON and 4-OHT-treated WT cells, as well as MYCN-OFF and mocktreated WT cells $(0 \mathrm{~h})$ (Supplementary Table 1$)$. Principal component analysis (PCA) revealed a clear separation of the MYCN-ON cells from the other conditions along the first principal component (PC) (Fig. 1c). Within the MYCN-ON samples, there was also a pronounced separation of individual time points, predominantly dictated by the second PC. This demonstrates that incubation with 4-OHT induces a specific and time-dependent transcriptional shift in MYCN-ON cells (Fig. 1c).

To assess the effect of MYCN activation on the RPE1 transcriptome, we performed gene set enrichment analysis (GSEA) using 30 publicly available $M Y C / M Y C N$ target gene sets. This revealed the expected enrichment $(\mathrm{FDR}<0.01)$ of MYC/MYCN targets in MYCN-ON cells at $24 \mathrm{~h}$ and onwards while this was not visible for the MYCN-OFF cells (Fig. 1d). In support thereof, RNA-sequencing and qPCR indeed revealed an upregulation of several selected MYCN target genes (e.g., ODC1, PTMA, WDR12, MDM2, NPM1, DKC1 1,8 (Supplementary Fig. 1a,b). When evaluating MYCN activity (translocation and signature activation) over time, the highest activity was noted at the $24 \mathrm{~h}$ time point, followed by sustained but attenuated activity at the later $48 \mathrm{~h}$ and $72 \mathrm{~h}$ time points (Fig. 1b, Supplementary Fig. 1c). This may be the result of a negative feedback loop as previously described $^{9}$, a notion supported by the lower MYCN transcript levels in MYCN-ON cells as compared to MYCNOFF cells at all time points (Supplementary Fig. 1d).

MYCN activation induces a p53-p21 driven growth attenuation. We noticed that MYCN-ON cells did not reach the same level of confluency $72 \mathrm{~h}$ p.i. as compared to controls (Fig. 2a). This pointed to an attenuation in cell proliferation, which was confirmed with a focus formation assay (P-value <0.01) (Fig. 2b). Quantitative immunofluorescence microscopy revealed that MYCN-ON cell populations harboured consistently fewer replicating (EdU-positive) cells than controls and progressively fewer cycling (Ki-67-positive) cells with time up to $72 \mathrm{~h}$ after MYCN induction (Fig. 2c-e). Caspase-3 and -7 activity was not significantly increased at any of the measured time points in MYCN-ON cells, suggesting there was no induction of apoptosis (Supplementary Fig. 2). DAPI-based cell cycle staging showed an increased fraction of cells in G1-phase and a significant decrease in G2-phase in MYCN-ON cells starting at $48 \mathrm{~h}$ p.i. (Fig. 2f).

To gain insight into the underlying cause of growth attenuation, we analysed accompanying changes in the transcriptome of MYCN-ON cells. Using gene set enrichment analysis (GSEA), we found enrichment for predicted p53 promotor-binding sites in the upregulated genes while the downregulated genes were enriched for FOXM1- and E2F1-binding targets, $48 \mathrm{~h}$ and $72 \mathrm{~h}$ after MYCN induction (Fig. 3a,b). Positive regulators of cell cycle transcription factors E2F1 and E2F2 were strongly downregulated after $24 \mathrm{~h}$ (Supplementary Fig. 3a). At all tested time points, transcriptional upregulation was found for Cyclin Dependent Kinase Inhibitor 1A $(C D K N 1 A)$, encoding the $\mathrm{p} 21$ protein and a direct target gene of $\mathrm{p} 53$. Induction of $\mathrm{p} 21$ by $\mathrm{p} 53$ is implicated in cell cycle arrest and senescence ${ }^{10,11}$ in accordance with the observed growth attenuation (Fig. 3c; Supplementary Fig. 3b). The p 1 and p16 cyclin-dependent kinase inhibitors are known to play key roles in the onset of cellular senescence as their simultaneous induction cooperatively blocks the activation of cyclin D kinases ${ }^{12}$. While cyclin dependent kinase inhibitor $2 \mathrm{~A}$ (CDKN2A encoding the p16 protein) expression was initially downregulated at $24 \mathrm{~h}$ p.i., we observed significant upregulation in MYCN-ON cells at the later 48 and $72 \mathrm{~h}$ p.i. time points. Of further interest, expression of cyclin dependent kinase 2 (CDK2) which has been reported to act as suppressor of oncogene induced senescence ${ }^{13}$ is significantly and progressively downregulated on a transcriptional level (Supplementary Fig. 3c). Quantitative immunofluorescence of MYCN-ON versus MYCN-OFF cells, revealed 
a
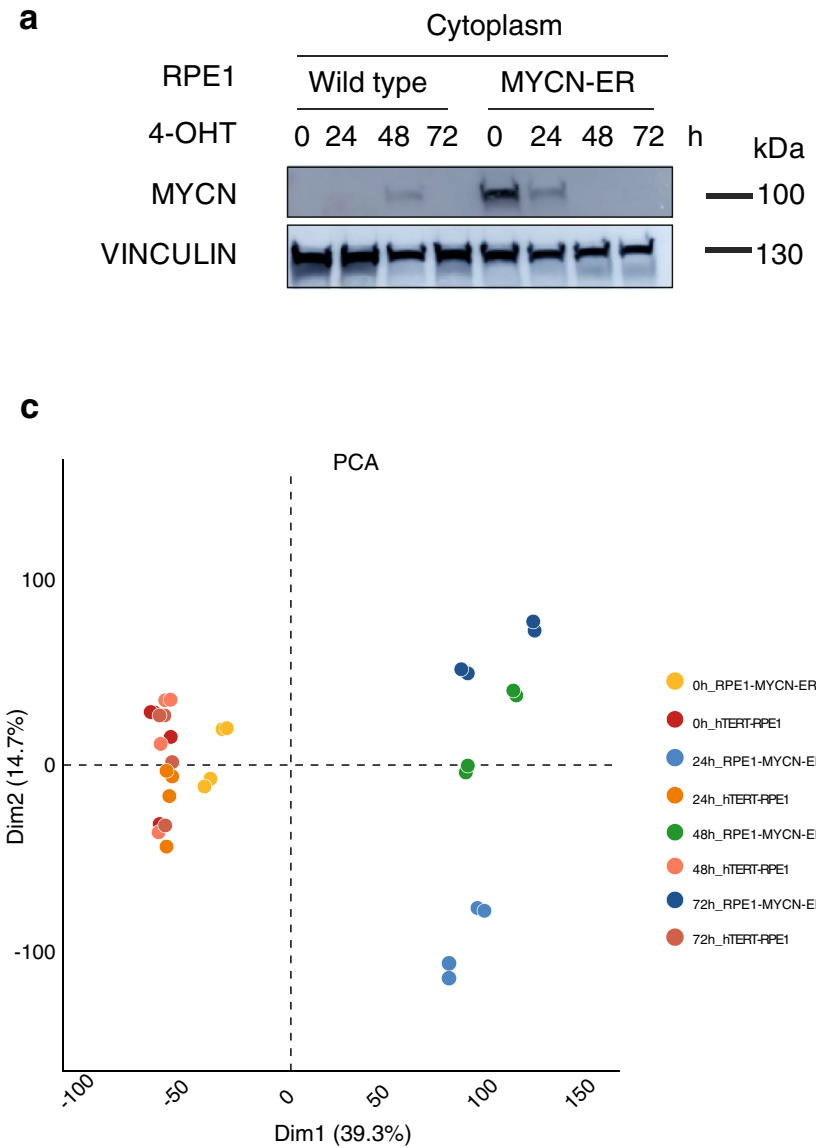

b

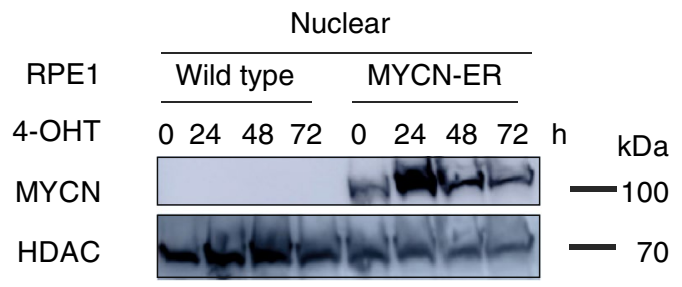

d

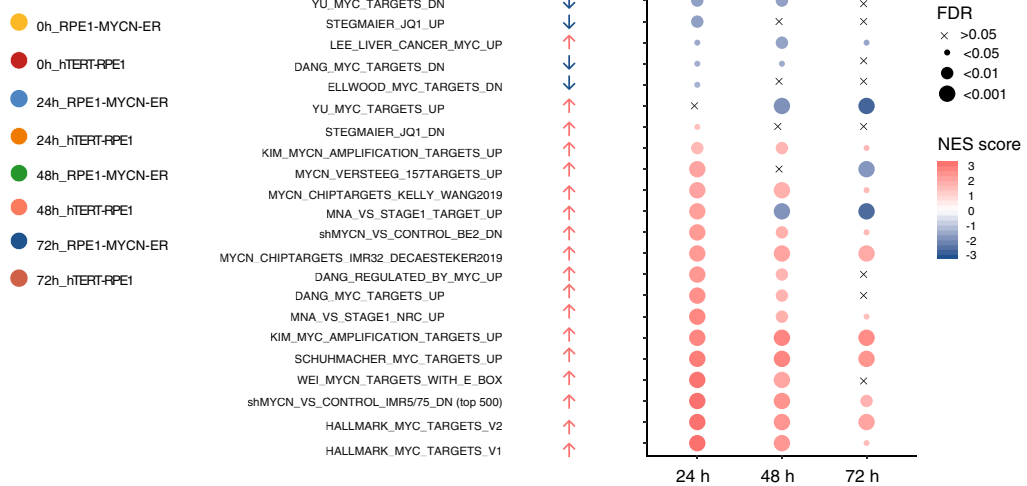

Figure 1. MYCN activation induces MYC/MYCN gene signatures with a specific and time-dependent transcriptional shift in MYCN-ON cells. Immunoblot of N-MYC nuclear (a) and cytoplasm (b) translocation in WT and RPE1-MYCN-ER cells upon 4-OHT treatment for 24, 48 and $72 \mathrm{~h}$. Vinculin was used as a cytoplasmic marker and loading control (a) while HDAC1 was used as a nuclear marker and loading control (b). (c) Principal component analysis and clustering with all samples, before differential analysis. All genes with a cpm greater than 1 in at least 6 samples are used in the analysis. Counts are normalized using edgeR and the data are rlog transformed. (d) 30 public available bona fide MYC/MYCN targets gene sets enriched in MYCN-ON cells $24 \mathrm{~h}, 48 \mathrm{~h}$ and $72 \mathrm{~h}$ p.i. compared to MYCN-OFF cells. Arrows represent the theoretically expected regulation of each signature in a positive (red) or negative (blue) manner for RPE1-MYCN-ER cells after MYCN activation. FDR value is depicted by dot size, GSEA normalised enrichment score (NES) is depicted by the colour of the dot. Western blots were cropped for clarity; uncropped images can be found in Supplementary Fig. 1e.

increased p53 and p 21 protein (Fig. 3d,e), in the absence of notable TP53 mRNA changes (Supplementary Fig. 3d). Deconvolution of nuclear marker intensities along the cell cycle revealed that p21 was much more abundant in G1-phase as compared to S or G2-phase (Supplementary Fig. 3e). This was already evident prior to MYCN induction (i.e., in MYCN-OFF), but became even more pronounced in MYCN-ON cells, suggesting this population to selectively enrich. In contrast, p53 protein levels increased in MYCN-ON cells independently of cell cycle status (Supplementary Fig. 3e,f).

Taken together, MYCN activation induces p53-p21 transcript and protein levels along with upregulation of p53 target genes and attenuated expression of FOXM1 and E2F target genes.

Induction of a pre-senescent transcriptional program in MYCN-ON cells. The combination of a marked growth attenuation and upregulation of $\mathrm{p} 53$ and $\mathrm{p} 21$ pathways, could be indicative for the induction of MYCN-triggered (oncogene-induced) senescence. To investigate this, we queried our RNA-seq dataset for a variety of senescence-related signatures (Fig. 4a) and observed a strong enrichment as of the $48 \mathrm{~h}$ time point after MYCN induction for the cell cycle arrest and senescence associated gene sets (Fig. 4a). In this context, the enrichment for a YAP/TAZ gene signature ${ }^{14,15}$ (Fig. 4a,b) is highly relevant given the reported downregulation of YAP proteins following replication-induced cellular senescence in IMR90 cells ${ }^{16}$. YAP/TAZ signaling has also been shown to control RRM2 transcription as a crucial effector in senescence control which is confirmed in our data showing a steep decline of RRM2 expression levels at $48 \mathrm{~h}$ p.i. ( $\mathrm{p}<0.0001$, ANOVA) (Fig. $4 \mathrm{c}, \mathrm{d})$. In further support of a senescence inducing gene signature, we observed downregulation of the senescence-associated biomarker LMNB1 both at the transcriptional ( $\mathrm{p}=6.51 \mathrm{e}-08$, ANOVA) and (at $72 \mathrm{~h}$ ) at protein level (Fig. 4c,e,f). In addition to the gene sets that showed a consistent up- or downregulation in MYCN-ON versus MYCN-OFF 
a MYCN-OFF

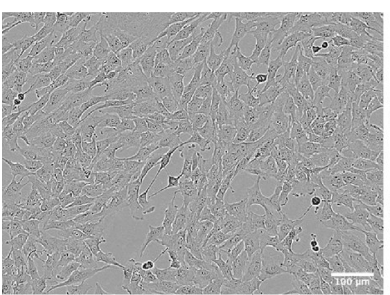

MYCN-ON

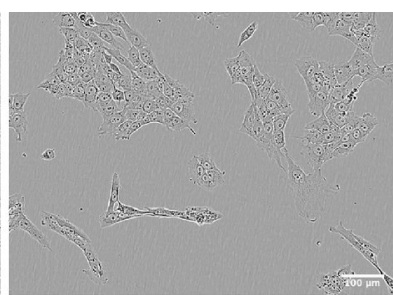

b

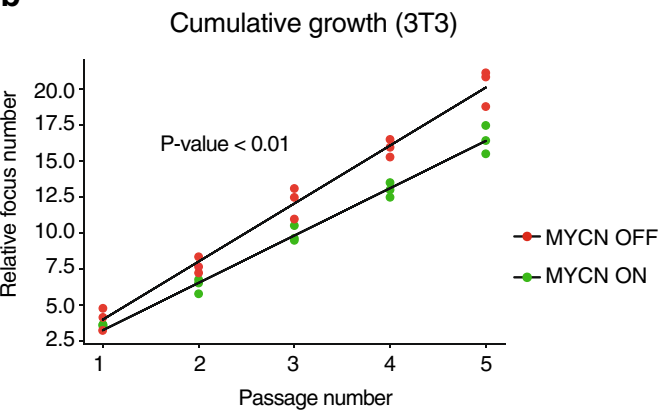

c

EdU

Ki-67

DAPI

MERGED
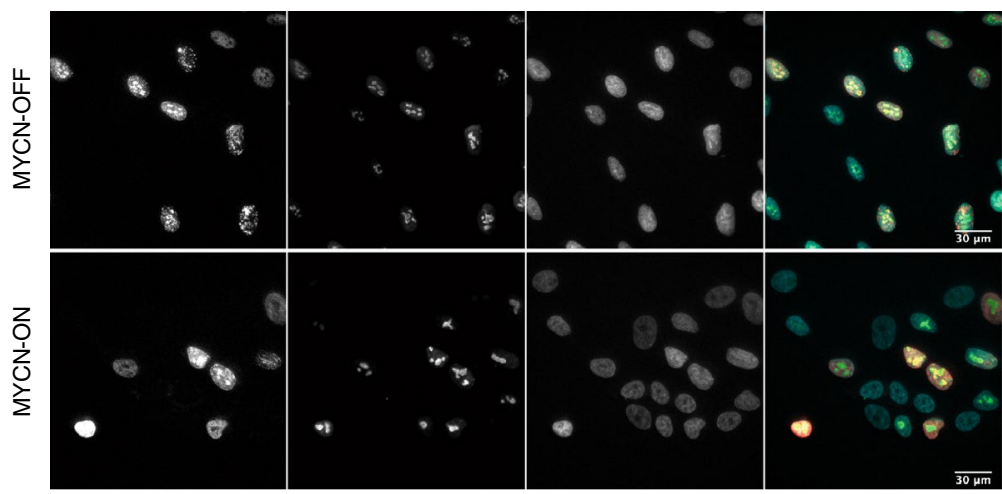

d

Cell proliferation (EdU integration)
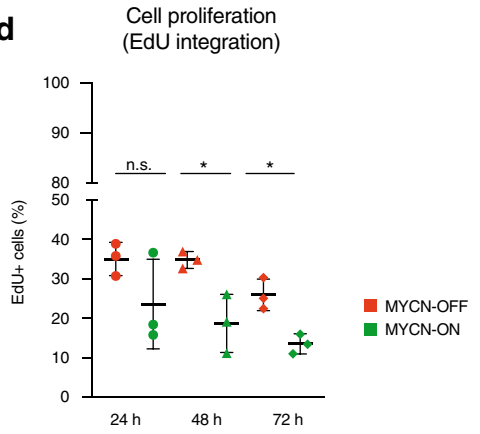
e Ki-67 positive fraction (cycling cells)

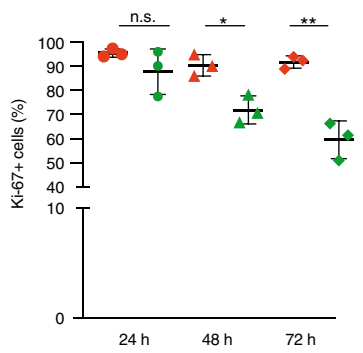

MYCN-OFF MYCN-ON

f
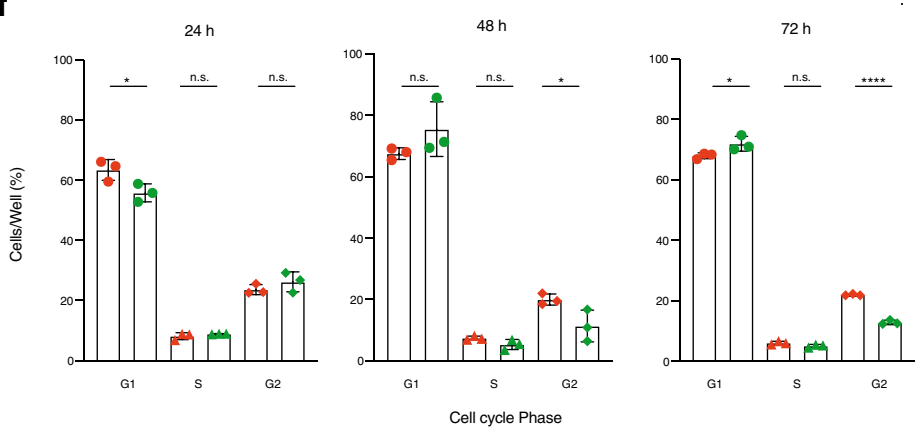

MYCN-OFF MYCN-ON

Figure 2. MYCN-ON cells undergo a slow-down in proliferation evidenced by reduced EdU incoporation and Ki-67 staining. (a) Phase contrast image of the corresponding cell line in both conditions $72 \mathrm{~h}$ p.i. (b) focus forming (NIH3T3) assay measuring the oncogenic potential of MYCN by calculating the cumulative increase in cell number. (c) Representative images of MYCN ON and OFF cells at $72 \mathrm{~h}$ p.i., after immunofluorescence staining for EdU (red), Ki-67 (green) and counterstaining with DAPI (cyan). Images were visualised and annotated in Fiji (v.2.0.0-rc-69/1.52p; http:// imagej.net/). (d) EdU incorporation immunofluorescence, expressed by the number of EdU positive nuclei relative to the total number of cells, in MYCN-ON and MYCN-OFF cells over time and (e) quantification of Ki-67, expressed by the number of Ki-67 positive nuclei relative to the total number of cells in MYCN-ON and MYCN-OFF cells over time. Error bars represent SD of the three biological replicates. (f) DAPI-based cell cycle staging upon MYCN activation. Statistical differences examined by unpaired Student's t-test. Error bars represent SD of the three biological replicates. (n.s. $=$ not significant, ${ }^{*}$ p-value $<0.05 ;{ }^{* *}$ p-value $<0,01 ;{ }^{* * *}$-value $<0.001$ ). Scale bar $=400 \mu \mathrm{m}$. 
cells, we also discovered signatures with bimodal distributions. In particular, two core senescence signatures ${ }^{17,18}$ $(\mathrm{p}<0.05)$ and a SASP-associated signature ${ }^{18}(\mathrm{p}<0.01)$ showed a marked drop at the $24 \mathrm{~h}$ time point in MYCNON cells, only to return to baseline (MYCN-OFF) levels at later time points. A similar observation was made for gene sets that become upregulated upon CDK4/CCND1 knockdown ( $\mathrm{p}<0.001)$ (Fig. 4a).

To dissect the different patterns of temporal gene expression changes following MYCN activation in more detail, we applied the elbow method for k-means clustering which allows the identification of an unbiased number of clusters in a given data set ${ }^{19}$. Using this approach, a total of 10 clusters was identified that captured the overall variation in the bulk RNA-seq data. Four clusters (3, 6, 7 and 8) were connected to MYCN signatures (Fig. 5a, Supplementary Fig. 4a, Supplementary Table 2), while cluster 10 was strongly enriched for gene targets of cell cycle master regulators FOXM1 and E2F. Three clusters (3, 7 and 8) consisted of gene sets associated with ribosome biogenesis, a well-known MYC $(\mathrm{N})$ driven process that is assumed to allow cells to cope with an increased demand for proteins required for cell growth and proliferation ${ }^{20}$ (Figs. 5a, S5a). These gene sets include EBNA1BP2 (EBP2) (cluster 3), which stabilizes MYC and promotes MYC-mediated rRNA synthesis and cell proliferation, as well as genes encoding proteins involved in ribosome RNA transcription $(P O L R 3 G)$, processing and maturation $(W D R 12, R R P 1 B)$ and ribosome assembly and shaping (MDN1) (cluster 8). Genes that composed cluster 7 exhibited increased expression $24 \mathrm{~h}$ after MYCN activation and robust downregulation at the $48 \mathrm{~h}$ time point. A prominent representative of this cluster was NPM1. NPM1 controls the impaired ribosome biogenesis checkpoint which becomes activated upon unscheduled MYC(N) driven ribosome production ${ }^{21}$. Checkpoint activation disables MDM2, leading to p53 stabilisation and p53 pathway activation, including p21 induction. Thus, the observed NPM1 overexpression during the very early phase of MYCN activation (24 h) suggests nucleolar stress is a driving factor in the p21-driven growth delay.

Finally, in order to gain insight into the critical transcriptional changes and overall heterogeneity of transcriptional induction of $C D K N 1 A$ and possible other early markers heralding the senescence transcriptome signature, we performed single cell RNA sequencing. As $C D K N 1 A$ was not yet significantly upregulated $16 \mathrm{~h}$ p.i., but was at $24 \mathrm{~h}$ p.i. (Supplementary Fig. 4b), we selected two time points around the CDKN1A inflection timepoint (22 h and $24 \mathrm{~h}$ ). Figure $5 \mathrm{~b}$ shows the inter-cellular heterogeneity of CDKN1A expression levels compared to untreated cells further supporting our immunohistochemistry staining (Fig. 3d, Supplementary Fig. 3f). When correlating CDKN1A expression levels within cell cycle stage, the higher expression levels were proportionally increased in all stages (Fig. 5c). This indicates that CDKN1A expression is increasing overall, already $24 \mathrm{~h}$ p.i.

Growth attenuation in MYCN-ON cells is accompanied by cytoplasmic granules and nucleolar coalescence. The above data prompted us to further investigate known senescence hallmarks. Unexpectedly, no evidence for $B$-galactosidase staining was found at any of the time points following MYCN activation studied for transcriptome profiling. Likewise, nuclear DAPI staining did not present evidence for senescenceassociated heterochromatin foci formation (SAHFs), another canonical phenotypic senescence marker ${ }^{22,23}$ (Supplementary Fig. 5a), and $\gamma \mathrm{H} 2 \mathrm{AX}$ and RPA32 staining did not reveal increased levels of DNA damage in MYCNON cells (Supplementary Fig. 5b,c). However, two obvious phenotypic changes were notable in MYCN-ON cells. First, we noticed a distinct nucleolar coalescence in the DAPI images of MYCN-ON cells. Quantitative immunofluorescence for fibrillarin (a protein component of C/D box small nucleolar ribonucleoproteins), confirmed this observation together with an overall increased nucleolar size (Fig. 6a,b), independently of the cell cycle status (Fig. 6c). Furthermore, a second marked change observed by transmission microscopy was a conspicuous cytoplasmic granularity in MYCN-ON cells (Fig. 6d). We reasoned that this might reflect a change in protein turnover as a consequence of the upregulated ribosomal biogenesis and nucleolar stress as also suggested by the strong NPM1 induction. However, neither staining for lysosomes, stress granules or P-bodies ${ }^{24,25}$ revealed colocalization with these granules, suggesting they do not represent typical relics of aberrant protein accrual and leaving their nature as yet undetermined (Supplementary Fig. 5d-g).

Taken together, we propose that unscheduled MYCN activation causes ribosome biogenesis and nucleolar stress in G2-phase, pre-priming cells for faltering in subsequent G1 and slowing down cell proliferation.

\section{Discussion}

Normal cells have tightly regulated signalling pathways that protect against insults such as unscheduled oncogene induction. Understanding how major oncogenic drivers such as MYCN activate and bypass such pathways during tumor initiation offers insights that may be of use for development of novel therapeutic strategies. Hence, we explored the use of immortalised neural crest-derived retinal pigment (RPE) cells to investigate the early effects of MYCN activation.

Unexpectedly, following MYCN activation, we observed attenuated cell growth without notable increased apoptosis but with a pronounced induction of $\mathrm{p} 53$ and $\mathrm{p} 21$. Attenuated proliferation was further accompanied by an increased number of cells in G1-phase and the induction of several previously reported senescence-induced gene signatures. While we observed a robust downregulation of LMNB1, other hallmarks of senescence (such as $\beta$-galactosidase activity and SAHFs) were not present, suggesting that cells were not undergoing full senescence. We also noticed progressive CDK2 transcriptional downregulation upon MYCN activation. CDK2 was previously shown to be essential to avoid MYC induced senescence in MEFs and required for sustained p21 and p16 induction ${ }^{13}$. The lack of strong p16 induction in the MYCN-ON cells might expmain the lack of full-blown induction of senescence in our model. The exact role and interaction of p21, p16 and CDK2 during MYCN activation remains to be explored, e.g. through CDK2 knock down and overexpression to further clarify the role of CDK2 in relation to p16 and p21 in the MYCN induced dynamic phenotypic changes in our model system.

Remarkably, MYCN-ON cells did show distinct phenotypic changes including nucleolar coalescence and cytoplasmic granularity, which aligned with transcriptional evidence for upregulation of ribosome biogenesis. 
ChEA_2016

a
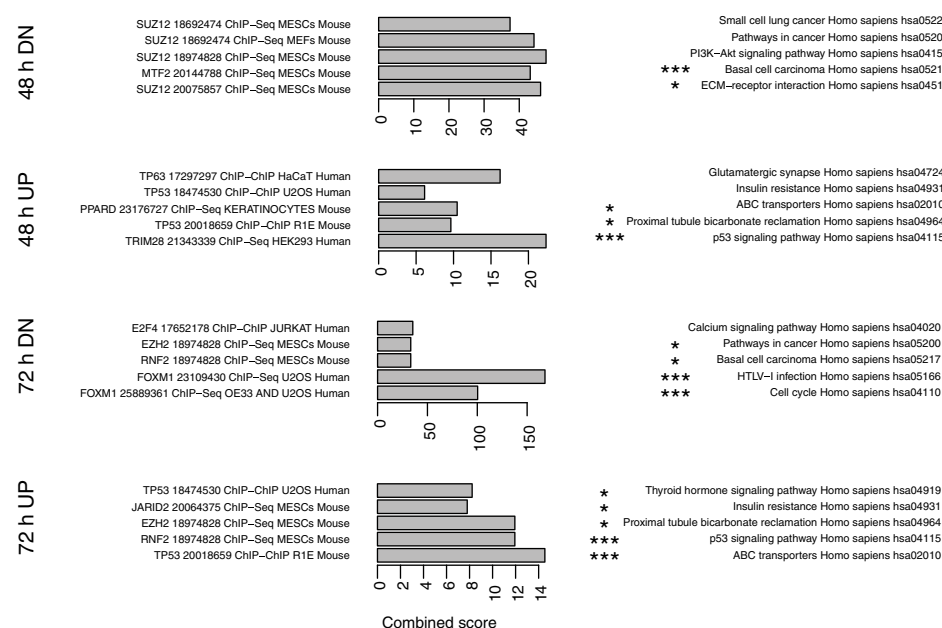

Combined score

b

FISCHER_MMB-FOXM1_TARGETS

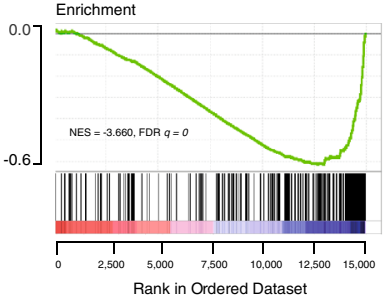

FISCHER_DIRECT_P53_TARGETS_META_ANALYSIS

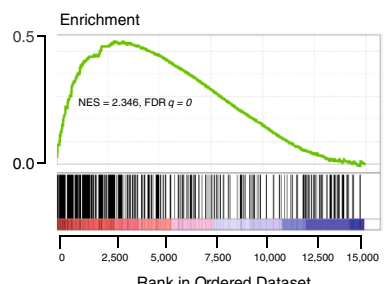

Rank in Ordered Dataset

d

p53

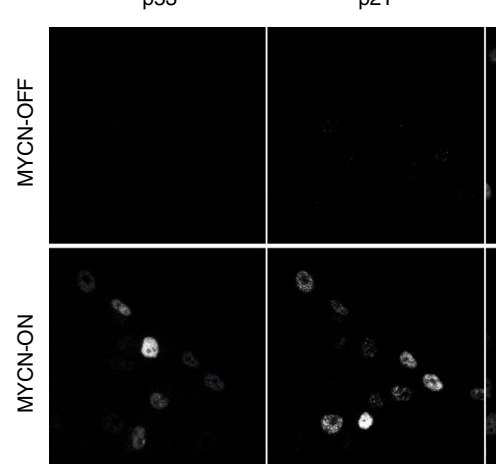

FISCHER_MMB-FOXM1_TARGETS

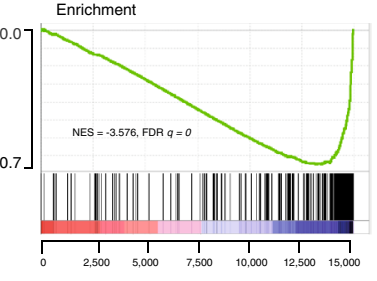

Rank in Ordered Dataset

FISCHER_DIRECT_P53_TARGETS_META_ANALYSIS

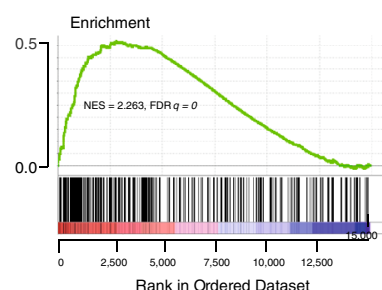

Rank in Ordered Dataset c

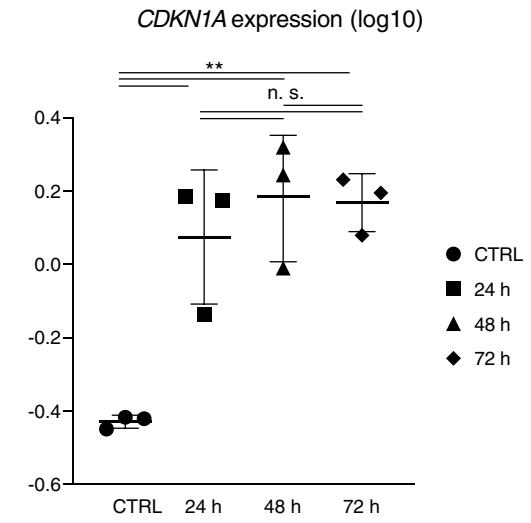

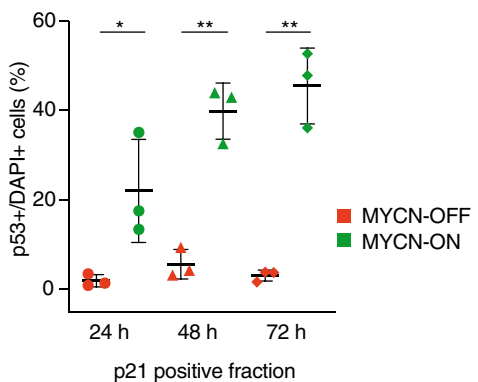

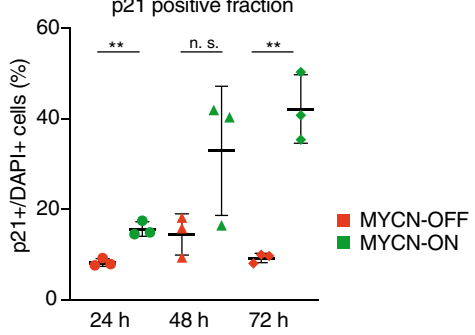


«Figure 3. MYCN activation triggers a p53-p21 driven growth attenuation. (a) Enrichr (https://maayanlab. cloud/Enrichr/) analysis shows enrichment for predicted p53 promotor binding sites in the upregulated genes and enrichment for FOXM1 and E2F1 binding in the downregulated genes $48 \mathrm{~h}$ and $72 \mathrm{~h}$ p.i. Combined Z-score is depicted based on the multiplication of log p value computed with Fisher exact test and the $\mathrm{z}$-score which is the deviation from the expected rank by the Fisher exact test. The top 5 ranked gene sets based on combined $\mathrm{z}$-score are depicted, which are all significant $(\mathrm{p}<0.05)$. Gene sets marked with an asterisk indicate significance upon multiple testing correction with ${ }^{* *}$ adj p.val $<0.001$ and ${ }^{\star}$ adj p.val $<0.05$. (b) FOXM1 (top) and p53 (bottom) targets related gene sets are enriched in MYCN-ON $48 \mathrm{~h}$ p.i. (left) and $72 \mathrm{~h}$ p.i. (right) compared to untreated MYCN-OFF. (c) $\log 10$ CDKN1A mRNA levels $24 \mathrm{~h}, 48 \mathrm{~h}, 72 \mathrm{~h}$ p.i. Results are normalised to $18 s, H M B S$ and SDHA expression. (d) Representative images of MYCN-ON and OFF cells $72 \mathrm{~h}$ p.i., after immunofluorescence staining for p53 (green), p21 (red) and counterstaining with DAPI (cyan), with relative protein levels of p53 (top) and p 1 (bottom) in MYCN ON versus OFF, (e) as measured by immunofluorescence staining and quantitative image analysis. Images were visualised and annotated in Fiji (v.2.0.0-rc-69/1.52p; http://imagej.net/). Statistical differences examined by unpaired Student's t-test. Error bars represent SD of the three biological replicates (n.s. $=$ not significant, ${ }^{\star} \mathrm{p}$-value $<0.05 ;{ }^{* *} \mathrm{p}$-value $<0.01 ;{ }^{* *} \mathrm{p}$-value $<0.001$ ). Scale bar $=30 \mu \mathrm{m}$.

Enlarged and prominent nucleoli upon increased MYCN activity have been documented before ${ }^{26}$, but we now connect it to a transcriptional and phenotypic switch. We propose that ribosome biogenesis and the consequent nucleolar stress drives cells into what we propose to represent a pre-senescent state. Indeed, perturbed ribosomal biogenesis and nucleolar stress is known to drive nucleolar coalescence in senescent cells ${ }^{27-30}$ and nucleolar expansion has been observed in progeria cells as a result of increased translational throughput (ribosome biogenesis and protein synthesis) ${ }^{31}$. This could be mimicked by knockdown of $L M N A$ and overexpression of progerin, suggesting a general influence of the lamin network on nucleolar plasticity. It is therefore tempting to speculate that the loss of lamin B1 maybe thus also act upstream of this process.

Oncogene activation (such as MYCN) is well known to induce alterations in ribosome biogenesis and ultimately activate the IRBC (Impaired Ribosome Biogenesis Checkpoint) as ribosomal proteins interact with the central acidic domain of MDM2 and indirectly promotes the stabilization of $\mathrm{p} 53^{27}$. Interestingly, MYCN is implicated in upregulation of RPL (Ribosomal Protein Large) and RPS (Ribosomal Protein Small) proteins in neuroblastoma cells ${ }^{20}$. Based on our observations, we envision that the senescence-like response triggered by MYCN activation in RPE1-MYCN-ER cells reflects a dynamic response to the oncogenic stimulus. Intriguingly, despite robust induction of CDKN1A in the MYCN-ON cells, neither apoptosis nor complete growth arrest was observed, and cells continued to grow, albeit more slowly. In this context, the recent discovery of a so-called "Goldilocks" zone for p21 levels was observed to control the proliferation-senescence cell-fate decision after drug treatment ${ }^{32}$. Either a delayed or acute drug-induced p21 response led to senescence, while an intermediate p21 pulse enabled sustained proliferation. The cell-cycle dependent p21 overshoot that we witnessed in MYCN-ON cells may thus reflect an attempt to initiate cell cycle arrest, which was not completely successful, either by having insufficient intensity or improper timing.

In conclusion, we have identified a transient, pre-senescent state as early response to unscheduled MYCN activation. Further studies, including live cell imaging in this model and in vivo single cell analysis of early emerging hyperplastic lesions in MYC(N) transgenic animal tumor models can shed further light on the dynamic changes in gene activity and cellular processes that allow these cells to cope with the stress during early malignant transformation. These insights can also provide guidance towards critical dependencies and pathways as novel drug targets for MYC(N) driven malignancies, many of which are currently still associated with poor survival.

\section{Materials and methods}

Cell culture. The hTERT-immortalized MYCN retinal pigmented epithelial cell line (RPE1-MYCN-ER, kindly provided by Michael Hogarty, Children's Hospital of Philadelphia, Philadelphia, PA) carries a MYCN:ER expression construct that results in its constitutive expression as described by Maris et al. ${ }^{6}$. When 4 -OHT is present in the culture media, MycN:ER translocates to the nucleus where it is transcriptionally active. The wilde-type (WT) hTERT-immortalized retinal pigment epithelium (hTERT-RPE1, Clontech, Palo Alto, CA) and RPE1-MYCN-ER were cultured in DMEM/F-12 HEPES (31330-038; Invitrogen), supplemented with 10\% fetal bovine serum (FBS), 1\% penicillin/streptomycin (15140-122; Invitrogen), 1\% L-glutamin (25030-025; Invitrogen) and $0.01 \mathrm{mg} / \mathrm{ml}$ Hygromycin B (10687010; Invitrogen) according to standard procedures. Moreover, $1 \mu \mathrm{g} /$ $\mathrm{ml}$ puromycin (P7255; Sigma-Aldrich) was added to the RPE1-MYCN-ER medium composition as a selection marker for the 4-OHT inducible construct. Mycoplasma testing was done on a monthly basis. Using DNA sequencing of the KRAS coding sequence, we validated the presence of the p.G12_G13insAG variant reported by Di Nicolantonio et al. ${ }^{33}$ in RPE-hTERT cells. We classified this as a variant of unknown significance and noted very low expression levels (not shown). In keeping with this presumed lack of functional significance Benedict et al. ${ }^{34}$ could provide evidence for KRAS activation using a pull-down assay to specifically precipitate RAS-GTP. Therefore, we are confident that the RPE-hTERT immortalized cells used in our study are valid to test cellular and molecular effects of MYCN induction.

Cell seeding and treatment. To evaluate phenotypic and protein changes, RPE1-MYCN-ER cells were plated in 96 wells overnight in three biological replicates and treated with or without $400 \mathrm{nM} \mathrm{4-OHT} \mathrm{(H6278;}$ Sigma-Aldrich) dissolved in EtOH for $24 \mathrm{~h}$ (4000 cells per well), $48 \mathrm{~h}$ (2000 cells per well) and $72 \mathrm{~h}$ (1000 cells per well). For the detection of stress granules and P-bodies, a treatment with $0.5 \mathrm{mM} \mathrm{NaAsO}_{2}(\# \mathrm{~S} 7400$; Sigma- 
a<smiles>C1CC2CCC2C1</smiles>

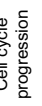

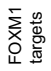

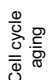

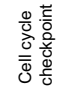

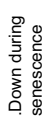

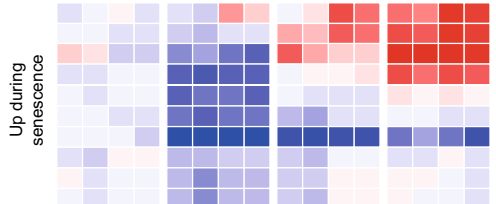

\begin{tabular}{l} 
D. \\
\hline
\end{tabular}

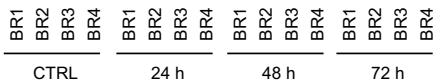

EGUCHI_CELL_CYCLE_RB1_TARGETS

BIOCARTA_DREAM_PATHWAY

FISCHER_DREAM_TARGETS

FISCHER_G2_M_CELL_CYCLE

CYCYCLE

WHITFIELD_CELL_CYCLE G2_M

MOLENAAR_TARGETS_OF_SiRNA_CCND1_AND_CDK4_DN

siFOXM1_vs_ctrl_10yfibroblasts_Down

siFOXM1_vs_ctrl_10yfibroblasts_MITOSIS_GO_DOWN

siFOXM1_vs_ctrl_10yfibroblasts_UP

SiFOXM1_vs_ctr__10yfibroblasts_MITOSIS_GO_UP

MMB-FOXM1_targets_87yfibroblasts_vs_neontal_DOWN

87yfibroblasts_vs_neonatal_MITOSIS_GO_DOWN

87yfibroblasts_vs_neonatal_DOWN

REACTOME P53 INDEPENDENT G1 S DNA DAMAGE CHECKPOINT REACTOME_SCFSKP2_MEDIATED_DEGRADATION_OF_P27_P21 TANG_SENESCENCE_TP53_TARGETS_DN

SiYAPTAZ_DN_SS2

DOWN LORAGINHO

SENESCENCE_CORE_SIGNATURE_DOWN_DEMARIO_2017

CHICAS_RB1_TARGETS_SENESCEN

CASELLA_SHARED_18_REDUCED_RNA_SENESC

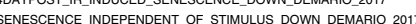

TANG SENESCENCE TP53 TARGETS UP

4DAYPOST IR INDUCEDSENESCENCE_UP DEMARIO 2017

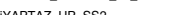

siRNA CCND1 AND CDK4 UP ***

DAYPOST IR_INDUCED_SENESCENCE_UP_DEMARIO_2017

SASP_SENESCENCE_UP_SiFOXM1_vs_control_10yfibroblast **

CASELLA_ONCOGENE_INDUCED_SENESCENCE_UP

SENESCENCE_CORE_SIGNATURE_UP_LORAGINHO *
SENESCENCE_CORE_SIGNATURE_UP_DEMARIO_2017 *

APOPTOSIS_BY_CDKN1A_VIA_TP53

WU_APOPTOSIS_BY_CDKNIA_NOT_VIA_TP

FSCHER_DIRECT_PS3_TARGETS_META_ANALYS

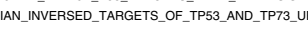

INGA_TP53_TARGETS

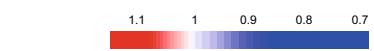

Signature Score b

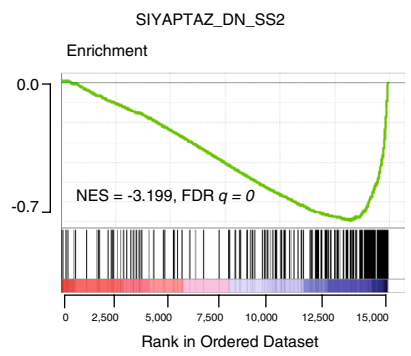

2DG_AND_YAPTAZ_DN

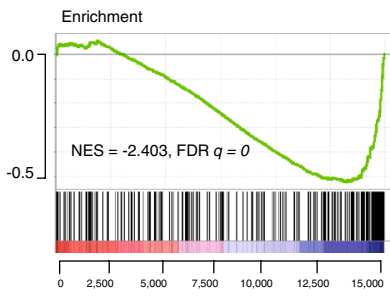

Rank in Ordered Dataset c

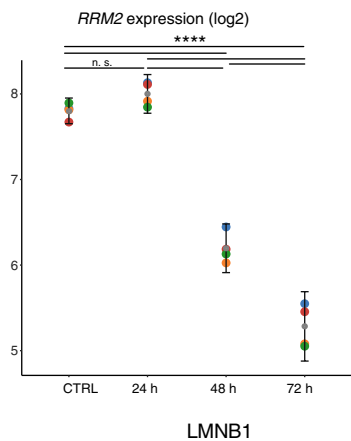

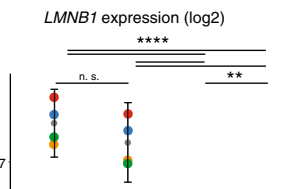

- Replicate 1

- Replicate 2

- Replicate 4

d

RRM2 expression $(\log 10)$
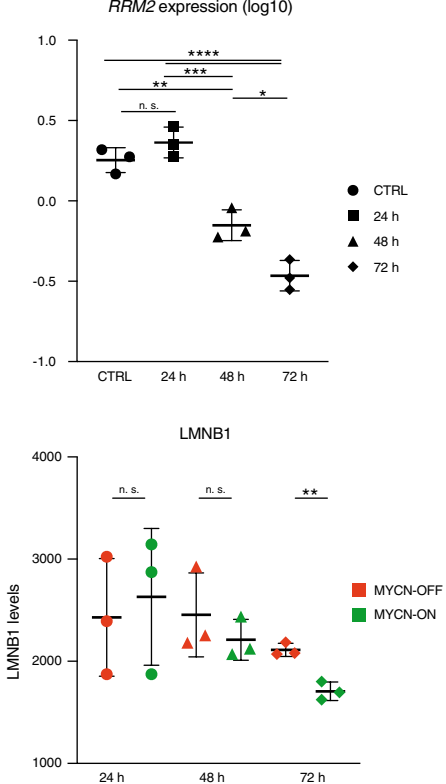
4Figure 4. Induction of MYCN triggers a senescence-like transcriptional program and phenotype in the absence of DNA damage. (a) Heatmap representing ranksum based signature scores for cell cycle, senescence, aging, FOXM1 and p53 associated gene sets over the different timepoints p.i. MYCN activation. All depicted gene sets were significantly enriched $(\mathrm{p}<0.05)$, those with adjusted $\mathrm{p}$-value below 0.05 are marked with an asterisk. BR = biological replicate. Images were generated using R Studio (v1.2.5019; https://www.rstudio.com). (b) MYCN activation correlates to inhibited dNTP metabolism as evidenced by GSEA for YAP/TAZ, which is enriched in MYCN-ON cells $72 \mathrm{~h}$ p.i. Enriched sets were used for visualization of gene set enrichment (v7.2 MSigDB; https://www.gsea-msigdb.org/gsea/index.jsp). (c) Log2 RRM2 and LMNB1 mRNA levels at 24 h, $48 \mathrm{~h}, 72 \mathrm{~h}$ p.i. of MYCN activation. ANOVA statistical analysis followed by a post-hoc Tukey's test for multiple comparisons. Error bars represent 95\% CI of the four biological replicates. (d) $\log 10$ RRM2 mRNA levels $24 \mathrm{~h}$, $48 \mathrm{~h}, 72 \mathrm{~h}$ p.i. Results are normalised to $18 \mathrm{~s}, H M B S$ and SDHA expression. Statistical differences examined by ANOVA statistical analysis followed by a post-hoc Tukey's test for multiple comparisons. (e) Representative images of MYCN ON and OFF cells at $72 \mathrm{~h}$ p.i., after immunofluorescence staining for LMNB1 (green) and counterstaining with DAPI (cyan). Images were visualised and annotated in Fiji (v.2.0.0-rc-69/1.52p; http:// imagej.net/). (f) Quantification of lamin B1 average nuclear intensity in MYCN ON and OFF cells. Statistical differences examined by unpaired Student's t-test. Error bars represent the SD of the three biological replicates. (n.s. $=$ not significant, ${ }^{\star}$ p-value $<0.05 ;{ }^{\star *}$ p-value $<0.01 ;{ }^{* *}$ p-value $\left.<0.001\right)$. Scale bar $=30 \mu \mathrm{m}$.

Aldrich) for $1 \mathrm{~h}$ served as positive control ${ }^{35}$. For DNA damage detection, the positive control consisted of a treatment with $1 \mu \mathrm{M}$ triapine (3-AP) (\#2251; Tocris) for $72 \mathrm{~h}^{36}$.

Focus formation (NIH-3T3) assay. To quantify the impact of the oncogene MYCN on the proliferation rate over time and several passages, a NIH-3T3-assay was applied as previously described by Todaro et al. ${ }^{37}$. In brief, cells with an inducible MYCN OFF/ON system, respectively either maintained in control conditions (EtOH) or induced MYCN (4-OHT), were plated in T25 flasks at a density of 0,15 million cells. The cumulative increase in cell number was calculated according to the formula $\log (\mathrm{Nf} / \mathrm{Ni}) / \log 2$, where $\mathrm{Ni}$ and $\mathrm{Nf}$ are the initial and final numbers of cells plated and counted after 3 or 4 days respectively.

Nuclear and cytoplasmic fractionation. The protocol was conducted as described earlier ${ }^{38}$. In brief, cells were grown as monolayers in T75 flasks, scraped from culture flasks and incubated in $1.5 \mathrm{~mL}$ of Harvest

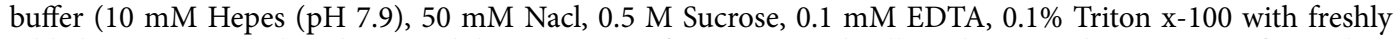
added protease and phosphatase inhibitors) at $4{ }^{\circ} \mathrm{C}$ for $15 \mathrm{~min}$ and collected in $1.5 \mathrm{ml}$ micro-centrifuge tubes. After centrifugation $(2000 \times g$ for $10 \mathrm{~min})$, supernatant (cytoplasmic fraction) was collected from each sample and subjected to $13,000 \times g$ for another $10 \mathrm{~min}$ to remove any nuclear contamination. The pellets were washed three times ( 3 times $2000 \times g$ for $3 \mathrm{~min}$ ), with washing buffer ( $10 \mathrm{mM}$ Hepes ( $\mathrm{pH} 7.9), 10 \mathrm{mM} \mathrm{KCl}, 0.1 \mathrm{mM}$ EDTA, $0.1 \mathrm{mM}$ EGTA and freshly added protease and phosphatase inhibitors). Both the pellet and supernatant were boiled separately in Laemmli sample buffer with $\beta$-mercaptoethanol. Western blot was performed using PVDF membranes incubated with anti-vinculin (V9131, Sigma-Aldrich; 1:10,000 dilution) antibody as cytoplasmic loading control, anti-HDAC (sc-7872, Santa-Cruz; 1:500 dilution) as nuclear loading control and anti-MYCN as a target protein (sc-53993, Santa-Cruz; 1:1000 dilution) after blocking with 5\% non-fat milk in 1\% tween-20 in TBST. Membranes were washed with TBST followed by incubation with HRP-conjugated anti-rabbit (\#7074S, Cell Signalling Technologies; 1:4000 dilution) or anti-mouse secondary antibody (\#7076S, Cell Signalling Technologies; 1:3000 dilution). After washing with TBST, imaging was performed using SuperSignal West DURA Extended Duration Substrate (34076X4; Thermo Scientific) and the Amersham Imager 680 (GE Healthcare).

Real-time quantitative PCR. Total RNA was extracted using the mRNeasy kit (Qiagen) according to the manufacturer's instructions and concentration was determined with the Nanodrop (Thermo Scientific). cDNA synthesis was performed using the iScript Advanced cDNA synthesis kit from BioRad. PCR mix contained $5 \mathrm{ng}$ of cDNA, $2.5 \mu \mathrm{l}$ SsoAdvanced SYBR qPCR supermix (Bio-Rad) and $0.25 \mu \mathrm{l}$ forward and reverse primer (to a final concentration of $250 \mathrm{nM}$, IDT) and was analysed on the LC-480 device (Roche) for RT-qPCR cycling. Expression levels were normalized using expression data of 3 stable reference genes (18 s, HMBS, SDHA) and analysed using qBasePlus software (http://www.biogazelle.com). Relative abundance of ODC1 transcripts (forward: GAT GACTTTTGATAGTGAAGTTGAGTTGA; reverse: GGCACCGAATTTCACACTGA), CDKN1A transcripts (forward: CCTCATCCCGTGTTCTCCTTT; reverse: GTACCACCCAGCGGACAAGT), RRM2 transcripts (forward: AGGACATTCAGCACTGGGAA; reverse: CCATAGAAACAGCGGGCTTC were measured relative to $18 s$ (forward: TTCGGAACTGAGGCCATGAT; reverse: TTTCGCTCTGGTCCGTCTTG), HMBS (forward: GGCAATGCGGCTGCAA; reverse: GGGTACCCACGCGAATCAC) and SDHA (forward: TGGGAACAA GAGGGCATCTG; reverse: CCACCACTGCATCAAATTCATG) reference transcripts.

Caspase-Glo 3/7 assay reagent. Caspase-3/7 activity was detected using a luminometric assay kit (Caspase-Glo 3/7; Promega) according to the manufacturer's instructions. The reagent provides a proluminescent caspase- $3 / 7$ substrate, which contains the tetrapeptide sequence DEVD, in combination with luciferase and a cell-lysing agent. The addition of the Caspase-Glo 3/7 reagent directly to the assay well results in cell lysis, followed by caspase cleavage of the DEVD substrate, and the generation of luminescence. Luminescence detection was performed by means of a microplate reader (GloMax; Promega). 

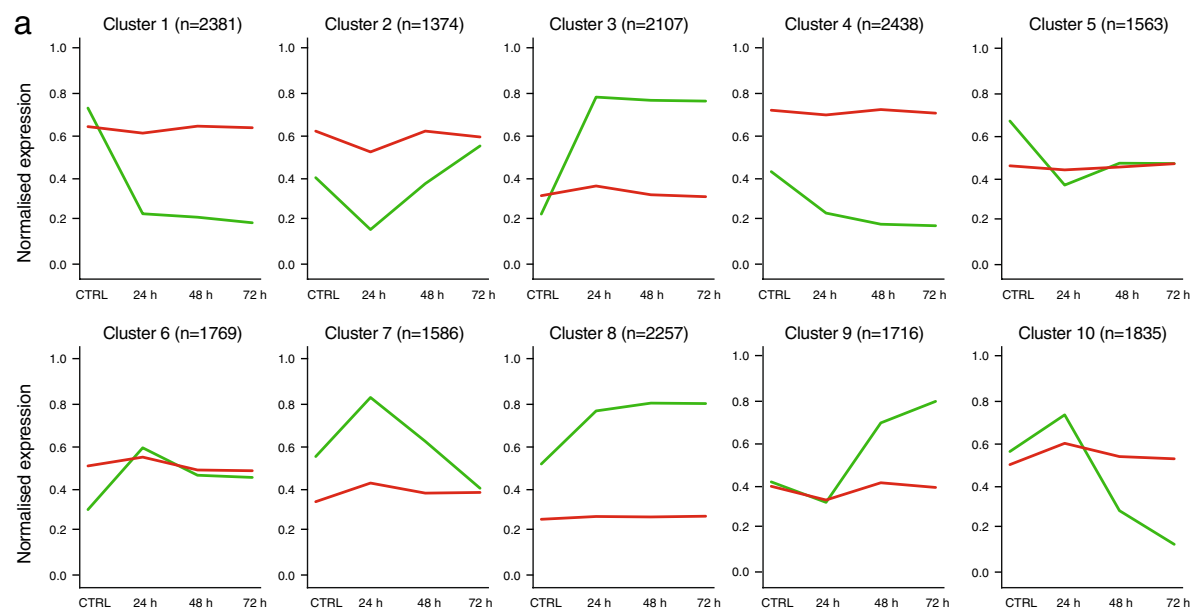

hTERT-RPE1

RPE1-MYCN-ER

b
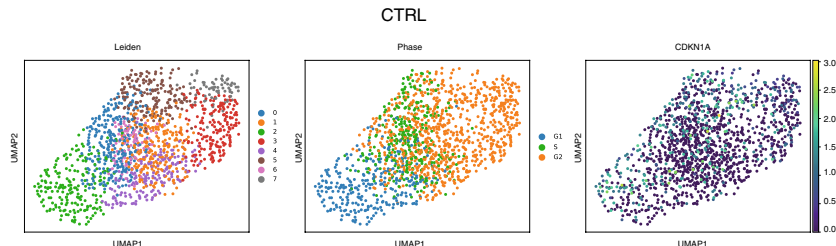

$22 \mathrm{~h}$
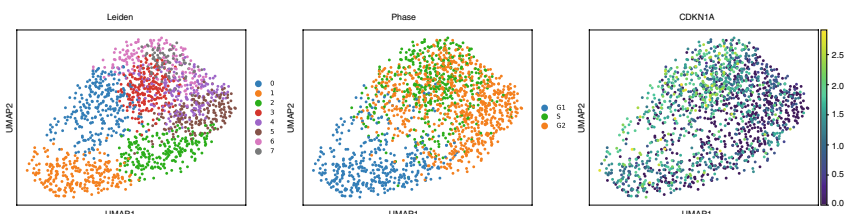

\begin{tabular}{|r|ccc|}
\hline & CTRL & $22 \mathrm{~h}$ & $24 \mathrm{~h}$ \\
G1 & 20.57 & 24.81 & 29.77 \\
S & 21.62 & 25.43 & 20.44 \\
G2 & 57.81 & 49.75 & 49.79 \\
\hline
\end{tabular}
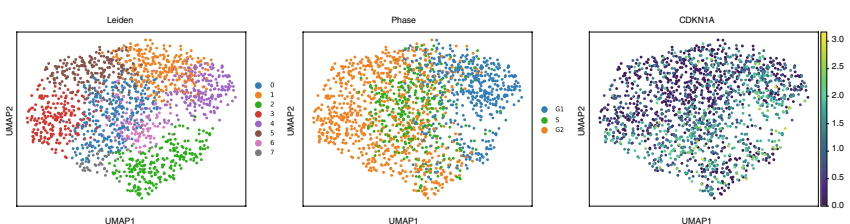

C

CDKN1A

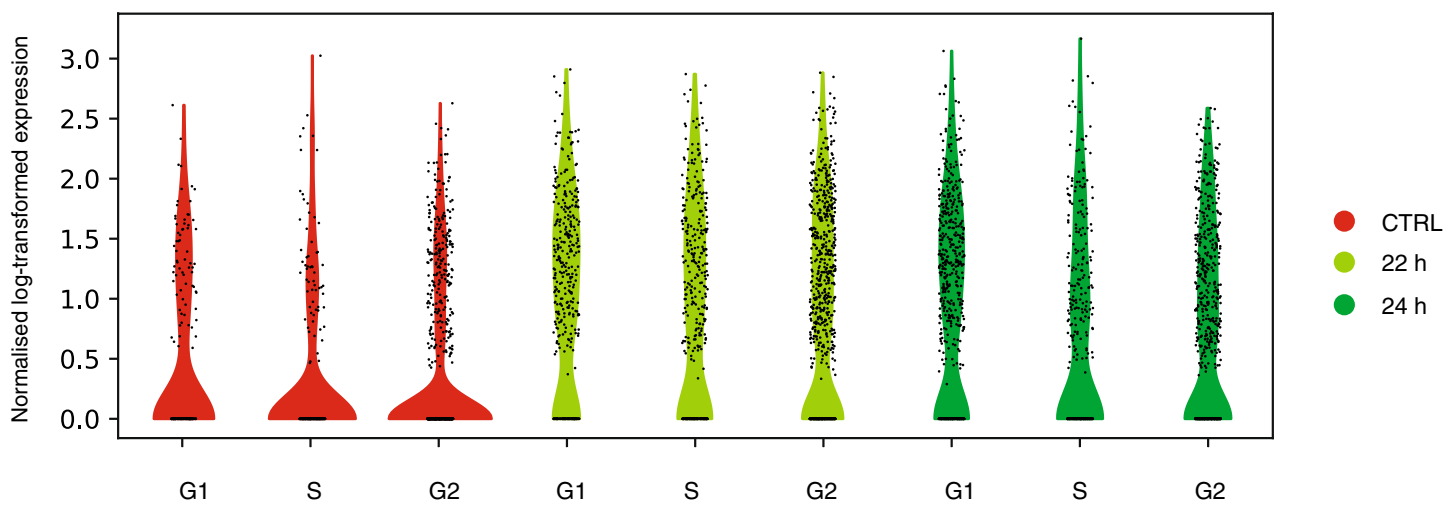

Figure 5. RNA-Sequencing indicates a heterogenous p21 response in MYCN-ON cells. (a) K-means clustering of all genes according to their average counts (quantile normalised) across replicates. (b) UMAP representation of leiden clusters, cell phase state and p 21 expression in the four treatment conditions separately. The table below shows the cell phase distribution for the treatments. (c) Normalised log-transformed expression CDKN1A expression within the single cell data set for 22 and $24 \mathrm{~h}$ p.i. Images were generated using a customized Python script (v 3.8; https://www.python.org/). 


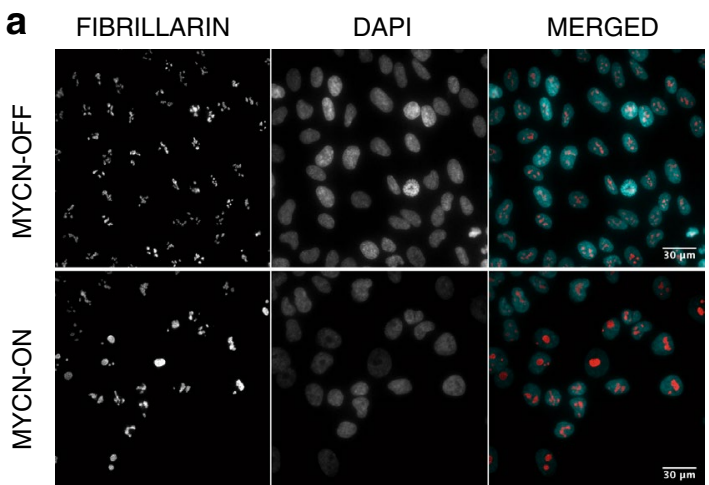

Fibrillarin/DAP

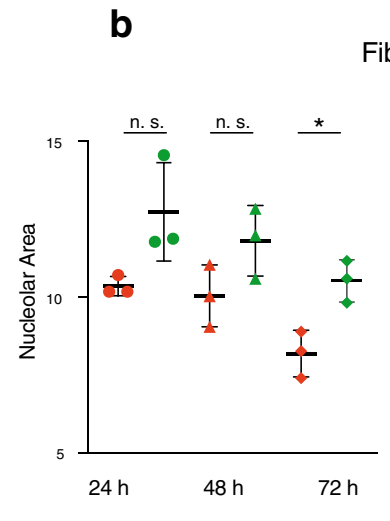

Fibrillarin

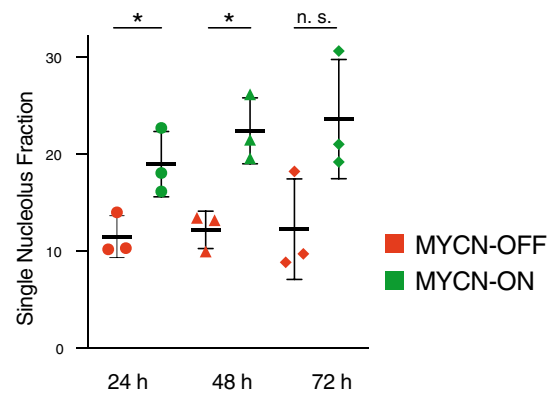

C
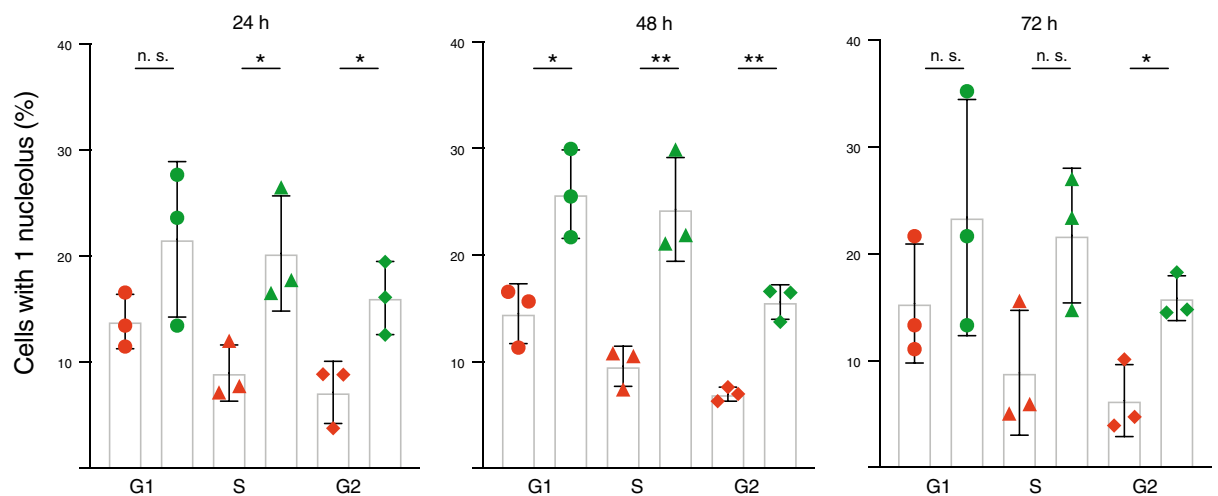

MYCN-OFF MYCN-ON

d
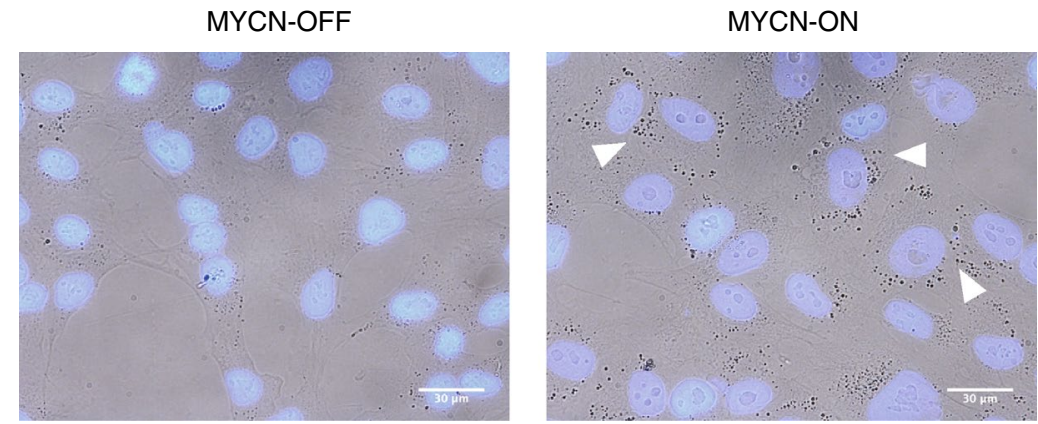

Figure 6. Nucleolar coalescence in MYCN-ON cells. (a) Representative images of MYCN-ON and OFF cells 72 p.i., after immunofluorescence staining for fibrillarin (red) (a protein component of C/D box small nucleolar ribonucleoproteins) counterstaining with DAPI (cyan). Images were visualised and annotated in Fiji (v.2.0.0-rc69/1.52p; http://imagej.net/). (b) Area of fibrillarin foci (left) and percentage of nuclei with 1 nucleolus (right) in MYCN ON versus OFF, as measured by immunofluorescence staining and quantitative image analysis. (c) DAPI-based cell cycle staging of cells with 1 single nucleolus. (d) Representative images of DAPI stained MYCN-ON and OFF cells show increase in nucleolar size and enhanced cytoplasmic granularity $72 \mathrm{~h} \mathrm{p.i.}$ Images were visualised and annotated in Fiji (v.2.0.0-rc-69/1.52p; http://imagej.net/). Statistical differences examined by unpaired Student's t-test. Error bars represent the SD of the three biological replicates. (n.s. $=$ not significant, ${ }^{*}$ p-value $<0.05 ;{ }^{* *}$ p-value $<0.01 ;{ }^{* *}$ p-value $\left.<0.001\right)$. Scale bar $=30 \mu \mathrm{m}$.

Immunofluorescence staining. RPE1-MYCN-ER cells were grown in black 96-well $\mu$ Clear plates (\# 655090, Greiner Bio-One) and fixed with $4 \%$ paraformaldehyde for $20 \mathrm{~min}$ at room temperature followed by washing $(3 \times 5 \mathrm{~min})$ with PBS (14190-094, Invitrogen). Subsequently, cells were permeabilized with $0.3 \%$ Triton X-100 ( $8 \mathrm{~min})$ and washed $(2 \times 5 \mathrm{~min})$, after which a blocking step was added with $50 \%$ FBS for 30 min and incubated with primary antibody diluted in 50\% FBS for $60 \mathrm{~min}$. After minimally 3 washing steps with PBS, plates were incubated with secondary antibody diluted in $50 \% \mathrm{FBS}$ for $60 \mathrm{~min}$, washed again, and counterstained with DAPI $(5 \mu \mathrm{g} / \mathrm{ml})$ for $20 \mathrm{~min}$. Primary antibodies were directed against p21 (F-5) (SC-6246, Santa Cruz Biotechnology Inc., 1:100 dilution), p53 (7F5) (\#2527 T, Cell Signalling Technology, 1:1600 dilution), Lamin B1 (ab16048, Abcam, 1:1000 dilution), Ki-67 (M7240) (M724029-2, Agilent, 1:400 dilution), $\gamma \mathrm{H} 2 \mathrm{AX}$ (phospho-S139) (ab2893, Abcam, 1:1000 dilution), RPA32/RPA2 (9H8) (ab2175, Abcam, 1:300 dilution), Fibrillarin (H-140) (SC-25397, Santa Cruz Biotechnology Inc., 1:50 dilution), DDX6 (200-192, Novus, 1:300 dilution) and 
G3BP1 (H00010146-M01, Abnova, 1:200 dilution). As secondary antibodies goat anti-rabbit IgG (H+L) Alexa Fluor 488 (A11034, Thermo Fisher, 1:400 dilution) and goat anti-mouse IgG (H+L) CY3 (A10521, Thermo Fisher, 1:400 dilution) were used. For cytoplasmic staining HCS CellMask ${ }^{\mathrm{Tx}}$ Red stain (H32712, Thermo Fisher) was added according to the manufacturer's instructions. After additional washing, the plates were maintained in $0.1 \% \mathrm{NaN}_{3} / \mathrm{PBS}$ and stored at $4{ }^{\circ} \mathrm{C}$ for microscopy.

Lysosome staining. Live cells were stained with $5 \mu \mathrm{g} / \mathrm{ml}$ of the nuclear dye Hoechst 33342 (\# H3570, Invitrogen) in medium at $37^{\circ} \mathrm{C}$ for $5 \mathrm{~min}$. After the plate was washed twice with PBS, $100 \mu \mathrm{l} /$ well $50 \mathrm{nM}$ Lysotrackerred DND-99 dye (\#L7528, Invitrogen) was added in medium at $37^{\circ} \mathrm{C}$ for $60 \mathrm{~min}$. Thereafter, medium was replenished and prepared for imaging.

EdU incorporation assay. EdU (5-ethynyl-2'-deoxyuridine), supplied within the Click-iT EdU Alexa Fluor 647 Imaging Kit (\#C10340, Thermo-Fisher Scientific, Waltham, MA, USA), was diluted in DMSO to a final concentration of $10 \mathrm{mM}$ and kept at $-20^{\circ} \mathrm{C}$. EdU was added to parallel cultures growing exponentially at $37^{\circ} \mathrm{C}$ in 96 well plates to final concentration of $20 \mu \mathrm{M}$ for $30 \mathrm{~min}$ to label the S-phase cells before fixation with $4 \%$ paraformaldehyde. The following steps of the Click-iT reaction were performed at room temperature. Additional antibodies were added as previously described in the immunofluorescence protocol. During the secondary antibody incubation step of the immunofluorescence protocol, the Click-iT kit azide and buffer additive were removed from $-20^{\circ} \mathrm{C}$ storage and allowed to thaw in a light-protected box. The buffer additive is stored as a $10 \times$ solution; once it is thawed, it must be diluted with $\mathrm{dH} 2 \mathrm{O}$ to make a working $1 \times$ solution. During the third PBS rinse after incubation with the secondary antibody, the reaction cocktail is made, adding the azide last. The reaction cocktail must be used within $15 \mathrm{~min}$ of creation. The amount of reaction cocktail needed was determined according to the Click-iT EdU Alexa Fluor 647 Imaging Kit as per manufacturer's instructions. Fixed cells were then incubated in the EdU cocktail for $30 \mathrm{~min}$ and rinsed three times in PBS for 5 min per rinse. The azide used was coupled to an Alexa Fluor 647 (red) fluorophore. Upon completion of the EdU Click-iT reaction, DAPI $(5 \mu \mathrm{g} / \mathrm{ml})$ was added for $20 \mathrm{~min}$ to the cells and after additional washing the plates were maintained in $0.1 \% \mathrm{NaN}_{3} / \mathrm{PBS}$ and stored at $4{ }^{\circ} \mathrm{C}$ for microscopy.

Microscopy. Image acquisition of fluorescently labelled cells (live or fixed) was performed using a Nikon Ti widefield fluorescence microscope, equipped with an Andor DU-885 X-266 camera. Per well, 16 fields were acquired in 4 channels $(405,488,561$ and $640 \mathrm{~nm}$ excitation) using a $40 \times$ dry $(\mathrm{NA}=0.95)$ objective. Live cells were imaged in DMEM/F-12 HEPES medium under environmental control $\left(37^{\circ} \mathrm{C}, 5 \% \mathrm{CO}_{2}\right)$.

Image processing and data analysis. Image processing was performed in FIJI (http://fiji.sc), a packaged version of ImageJ freeware (W.S. Rasband, USA. National Institutes of Health, Bethesda, Maryland, USA, http:// rsb.info.nih.gov/ij/, 1997-2014). Quantification of nuclear and spot signal intensities of (immuno-)stained cell cultures was done using a script specifically written for automated cell-based analysis (CellBlocks.ijm) ${ }^{39}$. In brief, the image analysis pipeline relies on the segmentation of nuclei, cells and intracellular spots and subsequent feature (number, intensity, shape...) extraction for all regions of interest. Downstream data analysis was performed in R Studio and graphs were generated by GraphPad Prism (version 8). To identify numbers (and percentages) of marker-positive and -negative cell nuclei, a manual threshold was set on the intensity values of the marker of interest. For cell cycle staging, we plotted the integrated nuclear signal intensity of the DAPI channel ${ }^{40}$. The results shown are averages (with standard deviation interval, tested by unpaired $t$-test) of a minimum of three independent experiments. Significance levels were indicated as follows: $p<0.05\left(^{*}\right), p<0.01\left(^{* *}\right)$, and $p<0.001$ $(* * *)$.

Bulk RNA-sequencing. To evaluate transcriptomic changes RPE1-MYCN-ER and WT hTERT-RPE1 cells were plated in T75 flasks overnight in four biological replicates and treated with or without $400 \mathrm{nM} 4$-OHT (H6278; Sigma-Aldrich) for $24 \mathrm{~h}, 48 \mathrm{~h}$ and $72 \mathrm{~h}$. Controls were supplied with the same amount of EtOH as was present in the treated cells and was always $\leq 0.5 \%$ of the total volume. Total RNA was extracted using the mRNeasy kit (Qiagen) according to the manufacturer's instructions. After RNA extraction, the concentration and quality of the total extracted RNA were checked by using the 'Quant-it ribogreen RNA assay' (Life Technologies, Grand Island, NY, USA) and the RNA 6000 Nano chip (Agilent Technologies, Santa Clara, CA, USA), respectively. Subsequently, $400 \mathrm{ng}$ of RNA was used to perform an Illumina sequencing library preparation using the QuantSeq 3' mRNA-seq Library Prep Kit (Lexogen, Vienna, Austria) according to the manufacturer's protocol. During library preparation, 14 PCR cycles were used. Libraries were quantified by qPCR, according to Illumina's protocol (Sequencing Library qPCR Quantification protocol guide, version February 2011). A High Sensitivity DNA chip (Agilent Technologies, Santa Clara, CA, US) was used to control the library's size distribution and quality. Sequencing was performed on a high throughput Illumina NextSeq 500 flow cell, generating 75 bp single reads. Per sample, on average, $7.5 \times 10^{6} \pm 1.6 \times 10^{6}$ reads were generated. First, these reads were trimmed using cutadapt $^{41}$ version 1.16 to remove the 'QuantSEQ FWD' adaptor sequence. The trimmed reads were mapped against the Homo sapiens GRCh38.89 reference genome using STAR ${ }^{42}$ version 2.6.0c. The RSEM ${ }^{43}$ software, version 1.3.1, was used to generate the count tables. To explore if the samples from different groups clustered together and to detect outlier samples, Principal Component Analyses (PCAs) on rlog transformed counts were performed using R, statistical computing software. Genes were only retained if they were expressed at counts per million (cpm) above one in at least four samples. Counts were normalized with the TMM method (R-package edgeR), followed by voom transformation and differential expression analysis using limma (R-package limma). Gene set enrichment analysis was performed on the ranked genes according to differential expression statisti- 
cal value ( $\mathrm{t}$ ) and corresponding heatmaps were made using a custom R script. Signature scores were conducted using a rank-scoring algorithm ${ }^{44}$. For the time-series analysis, all genes were clustered according to their average counts across replicates. Counts were quantile normalized across genes and across samples. Clusters were made with k-means method from the Python sklearn package (v0.22.2). To determine the ideal number of clusters, the elbow method was used ${ }^{19}$.

Single cell RNA-sequencing. Cells were harvested after treatment and washed two times with PBS with 0.04\% BSA (A7030; Sigma-Aldrich). The viability and the number of cells were measured with the Countess II (Invitrogen, Carlsbad, CA, USA). Samples were prepared for loading on the $10 \times$ Genomics Chromium microfluidics controller, as described in the Chromium Single Cell 3' protocol (10× Genomics, Pleasanton, CA, USA). Per sample, 5000 cells were loaded to end up with 2500 captured cells. During cDNA amplification, 12 PCR cycles were used. To add an index to the samples, another 12 PCR cycles were used. To check the final quality of the generated libraries, the samples were loaded on a Bioanalyzer High sensitivity chip (Agilent Technologies, Santa Clara, CA, US), and the quantity was checked with qPCR according to the Illumina protocol 'Sequencing Library qPCR Quantification protocol guide', version February 2011. Samples were pooled equimolarly and sequenced on a Hiseq4000 lane (Illumina, San Diego, CA, USA), generating paired-end reads of 150 base pairs. Afterward, the data were trimmed to paired-end reads of 28 and 91 base pairs. Single cell analysis was performed with the Python scanpy package (v1.5.1). Cells that reported less than 1000 genes were filtered, so were genes that were reported in only 1 or 2 cells. Cells were allowed to have up to $12 \%$ mitochondrial reads. For determining the differential expression of single cell genes, the Python package diffxpy (v0.7.4) was used. To calculate the bulk RPE1-MYCN-ER signatures in the single cell uniform manifold approximation and projection (UMAP) spaces, only genes were considered with an adjusted $p$-value $<0.01$. Single cell library sizes were adjusted to 10,000 and transformed by natural logarithm after addition of 1 . UMAPs with 3 components were produced by taking 10 neighbours in a 25 component PCA space. Cell phase was determined with the S and G2M gene sets used $\mathrm{in}^{45}$. In figures with all treatments together, cell phase was determined across all data, in figures were only one treatment is displayed, cell phase was determined for each cell only within that treatment data. Gene senescence signature scores were calculated as the number of genes that had expression in the cells. For the bulk RPE1-MYCN-ER signatures in the single cell, the average rank of the genes in the set was calculated. Full scripts can be found at https://github.com/dicaso/apetype/tree/master/examples/RPEMYCN.

Quantification and statistical analysis. Statistical analyses were performed in R Studio and statistical significance of differences between conditions for the immunofluorescence and caspase-glo experiments were determined by unpaired Student's t-test using GraphPad Prism (version 8). The ANOVA (analysis of variance) test followed by a post-hoc Tukey's test for multiple comparisons was used to determine differences in gene expression and signatures scores between 4 different groups with 3 (RT-qPCR) or 4 (RNA-Seq) biological replicates per condition. For RT-qPCR experiments, reference genes were excluded if GeNorm M value was greater than five and/or Coefficient of Variation greater than two, according to the qBase + software. All error bars represent the SD or $95 \% \mathrm{CI}$ of the three or four biological replicates. For statistical testing of the focus formation (NIH-3T3) assay, cumulative growth values were modelled using a linear model with time and treatment as fixed factors and an interaction term for both (Python package statsmodels). This model explained $99 \%$ of the observed variance and performed better than a restricted model omitting the interaction term for time and treatment (LR test P-value $<0.01$, interaction term P-value $<0.01$ in the more complex model). The details of quantification and statistical methods used are mentioned in the figure legends.

\section{Data availability}

The bulk and single-cell RNA-sequencing datasets generated during this study were deposited in the ArrayExpress database at EMBL-EBI EBI (http://www.ebi.ac.uk/arrayexpress) with accession numbers E-MTAB-9667 and E-MTAB-9689, respectively. All data generated or analyzed during this study are included in this published article and its supplementary information files.

Received: 25 March 2021; Accepted: 1 July 2021

Published online: 14 July 2021

\section{References}

1. Rickman, D. S., Schulte, J. H. \& Eilers, M. The expanding world of N-MYC-driven tumors. Cancer Discov. 8, 150-163. https://doi. org/10.1158/2159-8290.CD-17-0273 (2018).

2. Brady, S. W. et al. Pan-neuroblastoma analysis reveals age- and signature-associated driver alterations. Nat. Commun. 11, 5183. https://doi.org/10.1038/s41467-020-18987-4 (2020).

3. Hovestadt, V. et al. Medulloblastomics revisited: Biological and clinical insights from thousands of patients. Nat. Rev. Cancer. 20, 42-56. https://doi.org/10.1038/s41568-019-0223-8 (2020).

4. Nie, Z. et al. Dissecting transcriptional amplification by MYC. Elife 9, e52483. https://doi.org/10.7554/eLife.52483 (2020)

5. Baluapuri, A., Wolf, E. \& Eilers, M. Target gene-independent functions of MYC oncoproteins. Nat. Rev. Mol. Cell Biol. 21, $255-267$. https://doi.org/10.1038/s41580-020-0215-2 (2020).

6. Wang, Q. et al. ID2 expression is not associated with MYCN amplification or expression in human neuroblastomas. Cancer Res. 63, 1631-1635 (2003).

7. Oliynyk, G. et al. MYCN-enhanced oxidative and glycolytic metabolism reveals vulnerabilities for targeting neuroblastoma. iScience. 21, 188-204. https://doi.org/10.1016/j.isci.2019.10.020 (2019).

8. Westermann, F. et al. Distinct transcriptional MYCN/c-MYC activities are associated with spontaneous regression or malignant progression in neuroblastomas. Genome Biol. 9, R150. https://doi.org/10.1186/gb-2008-9-10-r150 (2008). 
9. Cole, K. A. et al. RNAi screen of the protein kinome identifies checkpoint kinase 1 (CHK1) as a therapeutic target in neuroblastoma. Proc. Natl. Acad. Sci. USA. 108, 3336-3341. https://doi.org/10.1073/pnas.1012351108 (2011).

10. Georgakilas, A. G., Martin, O. A. \& Bonner, W. M. p21: A two-faced genome guardian. Trends Mol. Med. 23, 310-319. https://doi. org/10.1016/j.molmed.2017.02.001 (2017).

11. Rufini, A., Tucci, P., Celardo, I. \& Melino, G. Senescence and aging: The critical roles of p53. Oncogene 32, 5129-5143. https://doi. org/10.1038/onc.2012.640 (2013).

12. Stein, G. H., Drullinger, L. F., Soulard, A. \& Dulić, V. Differential roles for cyclin-dependent kinase inhibitors p21 and p16 in the mechanisms of senescence and differentiation in human fibroblasts. Mol. Cell Biol. 19, 2109-2117. https://doi.org/10.1128/mcb. 19.3.2109 (1999)

13. Campaner, S. et al. Cdk2 suppresses cellular senescence induced by the c-myc oncogene. Nat. Cell Biol. 12, 54-59. https://doi.org/ $10.1038 /$ ncb2004 (2010).

14. Rajbhandari, P. et al. Cross-cohort analysis identifies a TEAD4-MYCN positive feedback loop as the core regulatory element of high-risk neuroblastoma. Cancer Discov. 8, 582-599. https://doi.org/10.1158/2159-8290.CD-16-0861 (2018).

15. Hiemer, S. E. et al. A YAP/TAZ-regulated molecular signature is associated with oral squamous cell carcinoma. Mol Cancer Res. 13, 957-968. https://doi.org/10.1158/1541-7786.MCR-14-0580 (2015).

16. Xie, Q. et al. YAP/TEAD-mediated transcription controls cellular senescence. Cancer Res. 73, 3615-3624. https://doi.org/10.1158/ 0008-5472.CAN-12-3793 (2013).

17. Hernandez-Segura, A. et al. Unmasking transcriptional heterogeneity in senescent cells. Curr. Biol. 27, 2652-2660. https://doi. org/10.1016/j.cub.2017.07.033 (2017).

18. Macedo, J. C. et al. FoxM1 repression during human aging leads to mitotic decline and aneuploidy-driven full senescence. Nat. Commun. 9, 2834. https://doi.org/10.1038/s41467-018-05258-6 (2018).

19. Ketchen, D. Jr. \& Shook, C. The application of cluster analysis in strategic management research: An analysis and critique. Strat. Manag. J. 17, 441-458. https://doi.org/10.1002/(SICI)1097-0266(199606)17:6\%3c441::AID-SMJ819\%3e3.0.CO;2-G (1996).

20. Boon, K. et al. $\mathrm{N}$-myc enhances the expression of a large set of genes functioning in ribosome biogenesis and protein synthesis. ЕМВО J. 20, 1383-1393. https://doi.org/10.1093/emboj/20.6.1383 (2001).

21. Hald, $\varnothing$. H. et al. Inhibitors of ribosome biogenesis repress the growth of MYCN-amplified neuroblastoma. Oncogene 38, 28002813. https://doi.org/10.1038/s41388-018-0611-7 (2019).

22. Salama, R., Sadaie, M., Hoare, M. \& Narita, M. Cellular senescence and its effector programs. Genes Dev. 28, 99-114. https://doi. org/10.1101/gad.235184.113 (2014).

23. Swanson, E. C., Manning, B., Zhang, H. \& Lawrence, J. B. Higher-order unfolding of satellite heterochromatin is a consistent and early event in cell senescence. J. Cell Biol. 203, 929-942. https://doi.org/10.1083/jcb.201306073 (2013).

24. Gorgoulis, V. et al. Cellular senescence: Defining a path forward. Cell 179, 813-827. https://doi.org/10.1016/j.cell.2019.10.005 (2019).

25. Anderson, P., Kedersha, N. \& Ivanov, P. Stress granules, P-bodies and cancer. Biochim. Biophys. Acta. 861-870, 2015. https://doi. org/10.1016/j.bbagrm.2014.11.009 (1849).

26. Kobayashi, C. et al. Enlarged and prominent nucleoli may be indicative of MYCN amplification: A study of neuroblastoma (Schwannian stroma-poor), undifferentiated/poorly differentiated subtype with high mitosis-karyorrhexis index. Cancer 103, 174-180. https://doi.org/10.1002/cncr.20717 (2005).

27. Turi, Z., Lacey, M., Mistrik, M. \& Moudry, P. Impaired ribosome biogenesis: Mechanisms and relevance to cancer and aging. Aging 11, 2512-2540. https://doi.org/10.18632/aging.101922 (2019).

28. Tiku, V. \& Antebi, A. Nucleolar function in lifespan regulation. Trends Cell Biol. 28, 662-672. https://doi.org/10.1016/j.tcb.2018. 03.007 (2018).

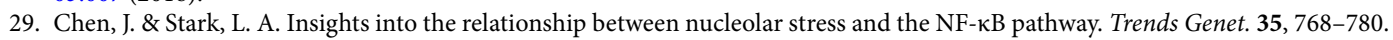
https://doi.org/10.1016/j.tig.2019.07.009 (2019).

30. Morlot, S. et al. Excessive rDNA transcription drives the disruption in nuclear homeostasis during entry into senescence in budding yeast. Cell Rep. 28, 408-422. https://doi.org/10.1016/j.celrep.2019.06.032 (2019).

31. Buchwalter, A. \& Hetzer, M. W. Nucleolar expansion and elevated protein translation in premature aging. Nat. Commun. 8, 328. https://doi.org/10.1038/s41467-017-00322-z (2017).

32. Hsu, C. H., Altschuler, S. J. \& Wu, L. F. Patterns of early p21 dynamics determine proliferation-senescence cell fate after chemotherapy. Cell 178, 361-373. https://doi.org/10.1016/j.cell.2019.05.041 (2019).

33. Di Nicolantonio, F. et al. Replacement of normal with mutant alleles in the genome of normal human cells unveils mutation-specific drug responses. Proc. Natl. Acad. Sci. USA. 105, 20864-20869. https://doi.org/10.1073/pnas.0808757105 (2008).

34. Benedict, B. et al. WAPL-dependent repair of damaged DNA replication forks underlies oncogene-induced loss of sister chromatid cohesion. Dev Cell. 52, 683-698. https://doi.org/10.1016/j.devcel.2020.01.024 (2020).

35. Matheny, T., Rao, B. S. \& Parker, R. Transcriptome-wide comparison of stress granules and P-bodies reveals that translation plays a major role in RNA partitioning. Mol. Cell Biol. 39, e00313-e319. https://doi.org/10.1128/MCB.00313-19 (2019).

36. Ishiguro, K. et al. Cataloging antineoplastic agents according to their effectiveness against platinum-resistant and platinum-sensitive ovarian carcinoma cell lines. J. Transl. Sci. 2, 117-124. https://doi.org/10.15761/JTS.1000127 (2016).

37. Todaro, G. J. \& Green, H. Quantitative studies of the growth of mouse embryo cells in culture and their development into established lines. J. Cell Biol. 17, 299-313. https://doi.org/10.1083/jcb.17.2.299 (1963).

38. Yue, M., Jiang, J., Gao, P., Liu, H. \& Qing, G. Oncogenic MYC activates a feedforward regulatory loop promoting essential amino acid metabolism and tumorigenesis. Cell Rep. 21, 3819-3832. https://doi.org/10.1016/j.celrep.2017.12.002 (2017)

39. De Vos, W. H., Van Neste, L., Dieriks, B., Joss, G. H. \& Van Oostveldt, P. High content image cytometry in the context of subnuclear organization. Cytometry A. 77, 64-75. https://doi.org/10.1002/cyto.a.20807 (2010).

40. Roukos, V., Pegoraro, G., Voss, T. C. \& Misteli, T. Cell cycle staging of individual cells by fluorescence microscopy. Nat. Protoc. 10, 334-348. https://doi.org/10.1038/nprot.2015.016 (2015).

41. Martin, M. Cutadapt removes adapter sequences from high-throughput sequencing reads. EMBnet J. https://doi.org/10.14806/ej. 17.1.200 (2011).

42. Dobin, A. et al. STAR: Ultrafast universal RNA-seq aligner. Bioinformatics 29, 15-21. https://doi.org/10.1093/bioinformatics/ bts635 (2013).

43. Li, B. \& Dewey, C. N. RSEM: Accurate transcript quantification from RNA-Seq data with or without a reference genome. $B M C$ Bioinform. 12, 323. https://doi.org/10.1186/1471-2105-12-323 (2011).

44. Fredlund, E., Ringnér, M., Maris, J. M. \& Påhlman, S. High Myc pathway activity and low stage of neuronal differentiation associate with poor outcome in neuroblastoma. Proc. Natl. Acad. Sci. USA 105, 14094-14099. https://doi.org/10.1073/pnas. 0804455105 (2008).

45. Pont, F., Tosolini, M. \& Fournié, J. J. Single-cell signature explorer for comprehensive visualization of single cell signatures across scRNA-seq datasets. Nucleic Acids Res. 47, e133. https://doi.org/10.1093/nar/gkz601 (2019). 


\section{Acknowledgements}

The authors would like to thank Dr. Michael D. Hogharty from the Division of Oncology at Children's Hospital of Philadelphia for providing the immortalized MYCN-ER-hTERT RPE1 cell line. We also acknowledge A. Eggermont, and F. De Vloed for technical assistance. We would like to thank E. De Meester and S. Decraene from NXTGNT Belgium for their expertise and assistance in the sequencing experiments of this study.

\section{Author contributions}

S.Z.: conceptualization, methodology, validation, formal analysis (real-time quantitative PCR, imaging data and statistical analysis), investigation, writing-original draft, writing-review and editing, visualization, project administration; S.V.: conceptualization, formal analysis (real-time quantitative pcr), writing-original draft, writing-review and editing, supervision, project administration; C.V.N.: conceptualization, methodology, software, formal analysis (single cell rna-sequencing data and statistical analysis), investigation, writing-review and editing, data curation, visualization, supervision; V.O.: software, formal analysis (single cell rna-sequencing), writing-review and editing; J.V.L.: investigation, writing—review and editing; M.V.: software, formal analysis (imaging data and statistical analysis), data curation, visualization, writing - review and editing; J.V.D.M.: validation; L.M.M.: investigation, visualization, writing — review and editing; K.D.: supervision, writing-review and editing; L.T.: software, formal analysis (bulk RNA-sequencing data), writing-review and editing; D.D.: methodology, resources, supervision of NXTGNT, writing - review and editing; F.V.N.: conceptualization, methodology, resources, supervision of NXTGNT, writing - review and editing; M.D.H.: methodology, resources, writingreview and editing; B.D.: conceptualization, methodology, software, formal analysis (bulk RNA-sequencing data and statistical analysis), data curation, writing - original draft, writing - review and editing, visualization, supervision; W.H.D.V.: conceptualization, methodology, software, formal analysis (imaging data and statistical analysis), resources, writing_original draft, writing_review and editing, supervision, project administration, funding acquisition; F.S.: conceptualization, resources, writing-original draft, writing-review and editing, supervision, project administration, funding acquisition.

\section{Funding}

This research was supported by 'Kom op tegen Kanker' (Stand up to Cancer), the Flemish Cancer Society (research grant to F. Speleman); Kinderkankerfonds (the non-profit childhood cancer foundation under Belgian law, Research grant to F. Speleman); the young investigator proof-of-concept project funded by the Cancer Research Institute Ghent (CRIG); Olivia Hendrickx Research Fund vzw. The following authors B. Decaesteker $(1238420 \mathrm{~N})$, S. Vanhauwaert (12U4718N), C. Van Neste (12N6917N), M. Verschuuren (11ZF116N) and K. Durinck (12Q8319N) are supported by an FWO grant.

\section{Competing interests}

The authors declare no competing interests.

\section{Additional information}

Supplementary Information The online version contains supplementary material available at https://doi.org/ 10.1038/s41598-021-93863-9.

Correspondence and requests for materials should be addressed to F.S.

Reprints and permissions information is available at www.nature.com/reprints.

Publisher's note Springer Nature remains neutral with regard to jurisdictional claims in published maps and institutional affiliations.

(c) (i) Open Access This article is licensed under a Creative Commons Attribution 4.0 International License, which permits use, sharing, adaptation, distribution and reproduction in any medium or format, as long as you give appropriate credit to the original author(s) and the source, provide a link to the Creative Commons licence, and indicate if changes were made. The images or other third party material in this article are included in the article's Creative Commons licence, unless indicated otherwise in a credit line to the material. If material is not included in the article's Creative Commons licence and your intended use is not permitted by statutory regulation or exceeds the permitted use, you will need to obtain permission directly from the copyright holder. To view a copy of this licence, visit http://creativecommons.org/licenses/by/4.0/.

(c) The Author(s) 2021 